\title{
Information State for Robust Control of Set-Valued Discrete Time
} Systems

\author{
John S. Baras ${ }^{1}$ and Nital S. Patel ${ }^{2}$ \\ Dept. of Electrical Engineering and Institute for Systems Research \\ University of Maryland, College Park, MD 20742
}

\begin{abstract}
We consider the problem of robust output feedback control of set-valued discrete time systems. Such systems appear frequently in applications such as process control. The aim is to motivate and obtain an efficient formulation of robust control problems and the resulting structure of the controller for such systems. This formulation, and the controller structure are obtained by taking small noise limits of the information state dynamics arising in a related risk-sensitive stochastic control problem. The necessary and sufficient conditions for solvability are also presented.
\end{abstract}

\section{Introduction}

Robust control addresses the problem of designing high performance controllers when there is uncertainty in the system to be controlled. The most demanding problems in robust control deal with model uncertainty. In most practical situations these types of uncertainties cannot be described as an additive disturbance; which is the typical model used in the literature on robust or $H_{\infty}$ control. This is more profound in the case of nonlinear systems, but even in the case of linear systems where the parameters are known to lie in certain numerical intervals (but their precise numerical values are not known). These considerations can be addressed if the problem of robust output feedback control is formulated in the context of systems modeled as

$$
\begin{aligned}
& x_{k+1} \in F\left(x_{k}, u_{k}\right) \\
& y_{k+1} \in G\left(x_{k}\right), k=0, \ldots, K-1
\end{aligned}
$$

where $F$ and $G$ are set-valued maps, and $x_{k} \in \mathbf{R}^{n}$ are the states, $u_{k} \in U \subset \boldsymbol{R}^{m}$ are the control inputs, and $y_{k} \in \mathbf{R}$ is the measured variable. Furthemore, $x_{0}$ is assumed to be 0 .

\footnotetext{
${ }^{1}$ Martin Marietta Chair in Systems Engineering.

e-mail: baras@isr.umd.edu

${ }^{2}$ e-mail: nsp@isr.umd.edu

This work was supported by the National Science Foundation Engineering Research Centers Program: NSFD CDR 8803012
}

The aim of this paper is to provide an appropriate formulation, and solution of the robust control problem for such systems. In particular, if we were given a regulated output $z$ along with the above dynamics, what would be an appropriate way to set up a control problem to minimize the influence of setvaluedness on $z$, while achieving certain performance objectives. Here, the set-valuedness is assumed to be due to bounded perturbations (additive and nonadditive) of a nominal single-valued system. We deal with the restricted case (i.e. having $x_{0}=0$ ), the results can be extended to the case of non-zero $x_{0}$.

We aim at obtaining the structure of the controller without invoking ad hoc assumptions on the structure, and without restricting to overtly simplified system classes. A key idea used in our approach to achieve this, following [5], is to study carefully the relationship between an associated risk-sensitive stochastic control problem and the dynamic game formulation of the robust control problem. The two are related via a small noise limiting procedure which helps to establish crisply the structure of the dynamic controller. The approach correctly constructs the information state of the controller and its dynamics; a key concept.

The main steps are as follows. In section 2, we consider a risk-sensitive stochastic control problem. We employ the idea in [5], where the small noise limit of a risk-sensitive stochastic control problem is taken to formally obtain an information state solution to the deterministic nonlinear $H_{\infty}$ control problem. In [5] an exponential cost function motivated from [3] was used, and small noise limits taken. We use the information state recursion derived from the stochastic control problem as the basis for the derivation of the dynamics of the controller for the deterministic problem. From our viewpoint, the stochastic control problem is entirely motivational, and we drop most of the assumptions associated with the small noise limit derivation when considering the deterministic problem in section 3 . The information state has a natural cost interpretation associated with it, and we postulate the minimization of this cost as a candidate formulation for the deterministic robust control prob- 
unknown, we could initialize the information state sequence $p_{0}$ as a smooth function weighing the initial states. We next define

$$
\begin{array}{r}
\Gamma_{0, k}^{u}\left(x_{0}\right) \triangleq\left\{x_{0, k} \in R_{0, k}^{n} \mid x_{l+1} \in F\left(x_{l}, u_{l}\right),\right. \\
l=0, \ldots, k-1\}
\end{array}
$$

where $\boldsymbol{R}_{0, k}^{n}=\left\{x_{0, k} \mid x_{l} \in \boldsymbol{R}^{n}, l=0, \ldots, k\right\}$, and

$$
\begin{array}{r}
\Gamma_{0, k}^{u, y}\left(x_{0}\right) \triangleq\left\{x_{0, k} \in \Gamma_{0, k}^{u}\left(x_{0}\right) \mid y_{l+1} \in G\left(x_{l}, u_{l}\right),\right. \\
l=0, \ldots, k-1\}
\end{array}
$$

Furthermore, we write $r, s \in \Gamma_{0, k}^{u}\left(x_{0}\right)$ for trajectories $r$ and $s$ such that $r \in \Gamma_{0, k}^{u}\left(x_{0}\right)$, and $s_{l+1} \in F\left(r_{l}, u_{l}\right)$, for $l=0, \ldots, k-1$, with $s_{0}=r_{0}=x_{0}$. We similary write $r, s \in \Gamma_{0, k}^{u, y}\left(x_{0}\right)$. Consider the information state recusion (6). By inspection, one obtains

$$
\begin{aligned}
p_{k}(x)=\sup _{r, s \in \Gamma_{0, k}^{u, y}(0)}\left\{\sum_{l=0}^{k-1} L\left(r_{l+1}, r_{l+1}-s_{l+1}, u_{l}\right)\right. \\
\left.-\frac{1}{2 \mu}\left|r_{l+1}-s_{l+1}\right|^{2} \mid r_{k}=x\right\}
\end{aligned}
$$

We now consider the following control problem for the system (4). Find a control policy $u \in U_{0, K-1}$, such that

$$
\sum_{k=0}^{K-1}\left\{L\left(r_{k+1}, r_{k+1}-s_{k+1}, u_{k}\right)-\frac{1}{2 \mu}\left|r_{k+1}-s_{k+1}\right|^{2}\right\} \leq 0
$$

for all trajectories $r, s \in \Gamma_{0, K}^{u}(0)$. Note that, if $r-s \in$ $l^{2}\left([0, K], \boldsymbol{R}^{n}\right)$, then the above guarantees that

$$
\frac{\left(\sum_{k=0}^{K-1} L\left(r_{k+1}, r_{k+1}-s_{k+1}, u_{k}\right)\right)^{\frac{1}{2}}}{\|r-s\|_{l^{2}}} \leq \frac{1}{\sqrt{2 \mu}}
$$

This immediately yields a method to set up robust control problems for the system $\Sigma$. Consider for example the following regulated output.

$$
z_{k+1}=h\left(x_{k+1}, u_{k}\right)
$$

where $x_{k}$ evolve via the dynamics (4). One could now consider attenuating the (Lipschitz) induced norm of $z$, (provided of course that $h$ is not, say, uniformly Lipschitz continuous in $x$ ) by defining $L$ as

$$
L(r, w, u)=|h(r, u)-h(r-w, u)|^{2}
$$

Such a problem is considered in detail in [1], and an example of its application to disturbance attenuation for a discontinuous system subject to parameter variations and additive noise is given in [2], where its performance is compared to an $H_{\infty}$ controller.

\subsection{Controller Structure}

Before, proceeding further, we define the following pairing, $(p, q) \triangleq \sup _{x \in \boldsymbol{R}^{n}}\{p(x)+q(x)\}$. We now state the solvability of the robust control problem in terms of a new cost function involving the information state $p_{k}$.
Lemma 3 For any $u \in U_{0, K-1}$, the closed loop system $\Sigma^{u}$ satisfies (7) on $[0, K]$, if and only if the information state $p_{k}$ satisfies

$$
\sup _{y_{1, k} \in \Delta^{u}(0)}\left\{\left(p_{k}, 0\right) \mid p_{0}=\delta_{\{0\}}\right\} \leq 0
$$

for all $k \in[0, K]$, where $\Delta^{u}(0)$ are all the measurements that can be generated by the closed loop system $\Sigma^{u}$.

Remark: The above result yields a separation principle, since one now deals solely with the information state system (6), where the information states $p$ plays the role of the states, and $y$ the role of disturbance. Hence, we have converted a partially observed system (4) to a fully observed (infinite dimensional) system (6), with the cost now given by the left hand side of equation (8). The problem now is to find a control policy $u \in U_{0, K-1}$ which achieves

$$
\inf _{u \in U_{0, k-1}} \sup _{y_{1, k} \in \Delta^{u}(0)}\left\{\left(p_{k}, 0\right) \mid p_{0}=\delta_{\{0\}}\right\}
$$

Such a policy will depend only on $p$, i.e. $u_{k}=u\left(p_{k}\right)$ and we call such policies information state feedback policies, and denote by $I_{0, K-1} \subset U_{0, K-1}$ the set of such policies.

Let $\mathcal{E}$ be the space in which $p$ lives. We define for a function $W: \mathcal{E} \rightarrow \boldsymbol{R}^{*}$

$$
\text { dom } W \triangleq\{p \in \mathcal{E} \mid W(p) \text { is finite }\}
$$

We employ dynamic programming to solve the problem. Define the value function by

$$
M_{k}(p)=\inf _{u \in U_{0, k-1}} \sup _{y \in \Delta_{1, k}^{u}(0)}\left\{\left(p_{k}, 0\right) \mid p_{0}=p\right\}
$$

for $k \in[0, K]$. The corresponding dynamic programming equation is

$$
M_{k}(p)=\inf _{u \in U} \sup _{\boldsymbol{y} \in \boldsymbol{R}}\left\{M_{k-1}(H(p, u, y))\right\}, k \in[1, K]
$$

with the initial condition $M_{0}(p)=(p, 0)$. Then, we obtain the following necessary and sufficient conditions for the solvability of the output feedback robust control problem.

Theorem 2 (Necessity) Assume that $\bar{u} \in O_{0, K-1}$ solves the finite time output feedback robust control problem with $x_{0}=0$. Then there exists a solution $M$ to the dynamic programming equation (9) such that $M_{k}\left(\delta_{0}\right)=0, M_{k}(p) \geq(p, 0), p \in \operatorname{dom} M_{k}, k \in[0, K]$.

Theorem 3 (Sufficiency) Assume there exists a solution $M$ to the dynamic programming equation (9) on some non-empty domain dom $M_{k}$, such that $\delta_{0} \in$ $\operatorname{dom} M_{k} M_{k}\left(\delta_{0}\right)=0, M_{k}(p) \geq(p, 0), k \in[0, K]$. Let $u^{*} \in I_{0, K-1}$ be a policy such that $u_{k}^{*}=\bar{u}_{K-k}^{*}\left(p_{k}\right)$, 
Then it can be shown that this satisfies the following dynamic programming equation

$$
\begin{aligned}
& V^{\mu, \varepsilon}(\sigma, k)=\inf _{u \in U_{k, k}} \mathbf{E}^{\dagger}\left[V ^ { \mu , \varepsilon } \left(\Sigma^{\mu, \varepsilon *}\left(u, y_{k+1}\right) \sigma,\right.\right. \\
&k+1)]
\end{aligned}
$$

for $k=0, \ldots, K-1$, where the infimizing control value $u_{k}(\sigma)$ solves the risk-sensitive control problem. It is clear that $u_{k}$ (the control value at time $k$ ) is a function only of (the information state) $\sigma_{k}^{\mu, \varepsilon}$ at time $k$. Hence, the policy is separated, and the information state contains all the relevant information required for control. We now take the small-noise limit of the information state recursion to construct an analogous filter for the deterministic robust control problem.

\subsection{Small Noise Limit}

We first define some spaces following [5]. For $\gamma \in$ $M \triangleq\left\{\gamma \in \boldsymbol{R}^{2} \mid \gamma_{1}>0, \gamma_{2} \geq 0\right\}$ define

$$
\begin{gathered}
\mathcal{D}^{\gamma} \triangleq\left\{\left.\tilde{p} \in C\left(\boldsymbol{R}^{n}\right)\left|\tilde{p}(x) \leq-\gamma_{1}\right| x\right|^{2}+\gamma_{2}\right\} \\
\mathcal{D} \triangleq\left\{\left.\tilde{p} \in C\left(\boldsymbol{R}^{n}\right)\left|\tilde{p}(x) \leq-\gamma_{1}\right| x\right|^{2}+\gamma_{2}\right. \\
\text { for some } \gamma \in M\}
\end{gathered}
$$

We equip these spaces with the topology of uniform convergence on compact subsets. Define $\Lambda^{\mu *}: \mathcal{D} \rightarrow \mathcal{D}$ by

$$
\begin{aligned}
& \Lambda^{\mu *}(u, y) \tilde{p}(z) \triangleq \\
& \sup _{\xi \in R^{n}}\left\{\tilde{p}(\xi)+\sup _{r \in F(\xi, u)}(L(z, z-r, u)-\right. \\
&\left.\left.\frac{1}{2 \mu}|z-r|^{2}\right)-\frac{1}{\mu} \inf _{s \in G(\xi)}\left(\frac{1}{2}|s|^{2}-s y\right)\right\}
\end{aligned}
$$

for $\tilde{p} \in \mathcal{D}$.

Then we have

Theorem 1

$$
\lim _{\varepsilon \rightarrow 0} \frac{\varepsilon}{\mu} \log \Sigma^{\mu, \varepsilon *}(u, y) e^{\frac{\mu}{\varepsilon} \tilde{p}}=\Lambda^{\mu *}(u, y) \tilde{p}
$$

in $\mathcal{D}$ uniformly on compact subsets of $U \times \boldsymbol{R} \times \mathcal{D}^{\gamma}$ for each $\gamma \in M$.

Proof: From (1) we have

$$
\begin{array}{r}
\frac{\varepsilon}{\mu} \log \Sigma^{\mu, \varepsilon *}(u, y) e^{\frac{\mu}{\epsilon} \bar{p}}(z)= \\
\frac{\varepsilon}{\mu} \log \int_{\boldsymbol{R}^{n}} \int_{F(\xi, u)} \int_{G(\xi)} \exp \frac{\mu}{\varepsilon}\left(\frac{-1}{2 \mu}|z-r|^{2}-\right. \\
\frac{n \varepsilon}{2 \mu} \log (2 \pi \varepsilon)+L(z, z-r, u)+\tilde{p}(\xi)+\frac{\varepsilon}{\mu} \log \chi(\xi, u)+ \\
\left.\quad \frac{\varepsilon}{\mu} \log \theta(\xi)-\frac{1}{\mu}\left[\frac{1}{2}|s|^{2}-s y\right]\right) d s d r d \xi
\end{array}
$$

Under the assumptions made on the system, a straightforward application of the Varadhan-Laplace lemma (Appendix) yields the result.

Remark: In particular, setting $\sigma^{\mu, \varepsilon}=e^{\frac{\mu}{\epsilon} \bar{p}}$ in equation (2), and employing the result of theorem 1, we obtain

$$
\begin{aligned}
& \tilde{p}_{k+1}(z)=\sup _{\xi \in \boldsymbol{R}^{n}}\left\{\tilde{p}_{k}(\xi)+\sup _{r \in F\left(\xi, u_{k}\right)}(\right. \\
& \left.L\left(z, z-r, u_{k}\right)-\frac{1}{2 \mu}|z-r|^{2}\right)- \\
& \left.\frac{1}{\mu} \inf _{\varepsilon \in G(\xi)}\left(\frac{1}{2}|s|^{2}-s y_{k+1}\right)\right\}
\end{aligned}
$$

for $k=0, \ldots, K-1$.

\section{Robust Control}

We now consider the deterministic system (corresponding to $\varepsilon \rightarrow 0$ ) defined by

$$
\Sigma\left\{\begin{array}{lll}
x_{k+1} & \in & F\left(x_{k}, u_{k}\right), x_{0}=0 \\
y_{k+1} & \in & G\left(x_{k}\right)
\end{array}\right.
$$

for $k=0, \ldots, K-1$. We assume that the system (4) satisfies the relevant assumptions of section 2 . Namely, that $F, G$ take on compact values with nonempty interior, and $u_{k} \in U$, with $U$ compact. We first simplify the information state recursion (3) for this case. Here it is assumed that we have access to the function $L$, which is tied to the particular kind of robust control problem being considered. More will be said about this in the next subsection. Note that we have forced $x_{0}=0$ here. The reason being that it simplifies the development. The general case can be dealt with in a similar manner [1].

We carry out the following change of variables in equation (3)

$$
\begin{aligned}
& p_{0}(x) \triangleq \tilde{p}_{0}(x) \\
& p_{k}(x) \triangleq \tilde{p}_{k}(x)-\frac{1}{2 \mu} \sum_{j=0}^{k-1}\left|y_{j+1}\right|^{2}, k=1, \ldots, K .
\end{aligned}
$$

Then equation (3) can be written as

$$
\begin{array}{r}
p_{k+1}(x)=\sup _{\xi \in \boldsymbol{R}^{n}}\left\{p_{k}(\xi)+\sup _{r \in F\left(\xi, u_{k}\right)}(\right. \\
\left.L\left(x, x-r, u_{k}\right)-\frac{1}{2 \mu}|x-r|^{2}\right)- \\
\left.\frac{1}{2 \mu} \inf _{s \in G(\xi)}\left(s-y_{k+1}\right)^{2}\right\}
\end{array}
$$

Using the convention that the supremum over an empty set is $-\infty$, we can place a natural restriction on $\xi$. Define

$$
\Omega(x, y, u) \triangleq\left\{\xi \in \boldsymbol{R}^{n} \mid x \in F(\xi, u) \text { and } y \in G(\xi)\right\}
$$

This just ensures that the values of $\xi$ are compatible with $x, u$ and $y$, given the dynamics (4). Then equation (5) can be written as

$$
\begin{array}{r}
p_{k+1}(x)=\sup _{\xi \in \Omega\left(x, y_{k+1}, u_{k}\right)}\left\{p_{k}(\xi)+\sup _{r \in F\left(\xi, u_{k}\right)}(\right. \\
\left.\left.L\left(x, x-r, u_{k}\right)-\frac{1}{2 \mu}|x-r|^{2}\right)\right\}
\end{array}
$$

or by (compactly) writing $H\left(p_{k}, y_{k+1}, u_{k}\right)(x)$ for the right hand side of (6) as

$$
\begin{aligned}
& p_{k+1}=H\left(p_{k}, y_{k+1}, u_{k}\right) \\
& p_{0}=\delta_{\{0\}}
\end{aligned}
$$

yielding the information state recursion for the deterministic system. Here, $\delta_{M}(\cdot): \boldsymbol{R}^{n} \rightarrow\{-\infty, 0\}$ is defined as $\delta_{M}(\xi)=0$ if $\xi \in M$, or else equal to $-\infty$ if $\xi \notin M$.

Remark: Since, $x_{0}=0$, we require $p_{0}=\delta_{\{0\}}$. This forces us to drop the requirement that $p$ be continuous for the deterministic case. However, if $x_{0}$ were 
Lemma 1 Under $\mathbf{P}^{\dagger}$, the random variables $\left\{y_{l}^{\varepsilon}\right\}$ are i.i.d. with density function $\phi^{\varepsilon}$.

Proof: Let $t \in \boldsymbol{R}$, and consider

$$
\begin{aligned}
\mathbf{P}^{\dagger}\left(y_{k}^{\varepsilon} \leq t \mid \mathcal{G}_{k-1}\right) & = \\
& \mathbf{E}^{\dagger}\left[I\left(y_{k}^{\varepsilon} \leq t\right) \mid \mathcal{G}_{k-1}\right] \\
& =\mathbf{E}^{u}\left[\Lambda_{k}^{\varepsilon} I\left(y_{k}^{\varepsilon} \leq t\right) \mid \mathcal{G}_{k-1}\right] / \mathbf{E}^{u}\left[\Lambda_{k} \mid \mathcal{G}_{k-1}\right]
\end{aligned}
$$

Now

$$
\begin{aligned}
& \mathbf{E}^{u}\left[\Lambda_{k}^{\varepsilon} \mid \mathcal{G}_{k-1}\right]= \\
& \Lambda_{k-1}^{\varepsilon} \theta\left(x_{k-1}^{\varepsilon}\right) \int_{R} \int_{G\left(x_{k-1}^{e}\right)} \phi^{\varepsilon}\left(v_{k}^{\varepsilon}+\xi\right) d \xi d v_{k}^{\varepsilon} \\
& \quad=\Lambda_{k-1}^{\varepsilon} \theta\left(x_{k-1}^{\varepsilon}\right) \int_{G\left(x_{k-1}^{e}\right)} \int_{R} \phi^{\varepsilon}\left(y_{k}^{\varepsilon}\right) d y_{k}^{\varepsilon} d \xi \\
& \quad \text { by changing the order of integration } \\
& \quad \text { and a change of variables. } \\
& \quad=\Lambda_{k-1}^{\varepsilon}
\end{aligned}
$$

and

$$
\begin{aligned}
& \mathbf{E}^{u}\left[\Lambda_{k}^{\varepsilon} I\left(y_{k}^{\varepsilon} \leq t\right) \mid \mathcal{G}_{k-1}\right] \\
& =\Lambda_{k-1}^{\varepsilon} \theta\left(x_{k-1}^{\varepsilon}\right) \int_{R} \int_{G\left(x_{k-1}^{e}\right)} I\left(y_{k}^{\varepsilon} \leq t\right) . \\
& =\Lambda_{k-1}^{\varepsilon} \theta\left(x_{k-1}^{\varepsilon}\right) \int_{G\left(x_{k-1}^{\varepsilon}\right)} \int_{R} I\left(y_{k}^{\varepsilon} \leq t\right) d \xi d v_{k}^{\varepsilon} \\
& \left.y_{k}^{\varepsilon}\right) d y_{k}^{\varepsilon} d \xi
\end{aligned}
$$

by changing the order of integration, and a change of variables.

$$
=\Lambda_{k-1}^{\varepsilon} \int_{-\infty}^{t} \phi^{\varepsilon}\left(y_{k}^{\varepsilon}\right) d y_{k}^{\varepsilon}
$$

The result follows.

It is clear that under $\mathbf{P}^{\dagger}, y_{l}^{\varepsilon}$, and $x_{l}^{\varepsilon}$ are independent. Furthermore, the existence of $\mathrm{P}^{\dagger}$ is guaranteed by Kolmogorov's extension theorem. In a similar manner, we define the inverse transformation relating $\mathbf{P}^{u}$ to $\mathbf{P}^{\dagger}$ as follows.

$$
\left.\frac{d \mathbf{P}^{u}}{d \mathbf{P}^{\dagger}}\right|_{\mathcal{G}_{k}}=\mathcal{Z}_{k}^{\varepsilon}=\Pi_{l=1}^{k} \Psi^{\varepsilon}\left(x_{l-1}^{\varepsilon}, y_{l}^{\varepsilon}\right)
$$

where

$$
\begin{aligned}
& \Psi^{\varepsilon}\left(x_{l-1}^{\varepsilon}, y_{l}^{\varepsilon}\right) \triangleq \theta\left(x_{l-1}^{\varepsilon}\right) \int_{G\left(x_{l-1}^{\varepsilon}\right)} \phi^{\varepsilon}\left(y_{l}^{\varepsilon}-\xi\right) d \xi / \phi^{\varepsilon}\left(y_{l}^{\varepsilon}\right) \\
&=\theta\left(x_{l-1}^{\varepsilon}\right) \int_{G\left(x_{l-1}^{\varepsilon}\right)} \exp \left(-\frac{1}{\varepsilon}\left(\frac{1}{2}|\xi|^{2}-\right.\right. \\
&\left.\left.-y_{l}^{\varepsilon} \xi\right)\right) d \xi
\end{aligned}
$$

\subsection{Information State}

Consider the space $L^{\infty}\left(\boldsymbol{R}^{n}\right)$ and its dual $L^{\infty *}\left(\boldsymbol{R}^{n}\right)$. We will denote the natural bilinear pairing between $L^{\infty}\left(\boldsymbol{R}^{n}\right)$ and $L^{\infty *}\left(\boldsymbol{R}^{n}\right)$ by $<\tau, \eta>$ for $\tau \in L^{\infty *}\left(\boldsymbol{R}^{n}\right)$, $\eta \in L^{\infty}\left(\boldsymbol{R}^{n}\right)$.

We define the information state process $\sigma_{k}^{\mu, \varepsilon} \in$ $L^{\infty *}\left(\boldsymbol{R}^{n}\right)$ by

$$
\begin{aligned}
& <\sigma_{k}^{\mu, \varepsilon}, \eta>= \\
& \quad \mathbf{E}^{\dagger}\left[\eta\left(x_{k}^{\varepsilon}\right) \exp \left(\frac{\mu}{\varepsilon} \sum_{l=1}^{k} L\left(x_{l}^{\varepsilon}, w_{l}^{\varepsilon}, u_{l-1}\right)\right) \mathcal{Z}_{k}^{\varepsilon} \mid \mathcal{Y}_{k}\right]
\end{aligned}
$$

for all test functions $\eta \in L^{\infty}\left(\boldsymbol{R}^{n}\right)$, for $k=1, \ldots, K$, with $\sigma_{0}^{\mu, \varepsilon}=\rho \in L^{1}\left(\boldsymbol{R}^{n}\right)$. We introduce the bounded linear operator $\Sigma^{\mu, \varepsilon}: L^{\infty}\left(\boldsymbol{R}^{n}\right) \rightarrow L^{\infty}\left(\boldsymbol{R}^{n}\right)$ defined by

$$
\begin{aligned}
& \Sigma^{\mu, \varepsilon}(u, y) \eta(\xi) \triangleq \\
& \quad \int_{\boldsymbol{R}^{n} \int_{F(\xi, u)}} \psi^{\varepsilon}(z-r) \exp \left(\frac{\mu}{\varepsilon} L(z, z-r, u)\right) . \\
& \eta(z) d r d z \Psi^{\varepsilon}(\xi, y) \chi(\xi, u)
\end{aligned}
$$

and its adjoint $\Sigma^{\mu, \epsilon *}: L^{\infty *}\left(\boldsymbol{R}^{n}\right) \rightarrow L^{\infty *}\left(\boldsymbol{R}^{n}\right)$ defined by

$$
\begin{aligned}
& \Sigma^{\mu, \varepsilon *}(u, y) \sigma(z) \triangleq \\
& \quad \int_{\boldsymbol{R}^{n} \int_{F(\xi, u)} \chi(\xi, u) \psi^{\varepsilon}(z-r) .} . \\
& \quad \exp \left(\frac{\mu}{\varepsilon} L(z, z-r, u)\right) \Psi^{\varepsilon}(\xi, y) \sigma(\xi) d r d \xi
\end{aligned}
$$

Lemma 2 The information state $\sigma_{k}^{\mu, \varepsilon}$ satisfies

$$
\left\{\begin{array}{l}
\sigma_{k}^{\mu, \varepsilon}=\Sigma^{\mu, \epsilon *}\left(u_{k-1}, y_{k}^{\varepsilon}\right) \sigma_{k-1}^{\mu, \varepsilon} \\
\sigma_{0}^{\mu, \varepsilon}=\rho
\end{array}\right.
$$

Proof:

$$
\begin{aligned}
<\sigma_{k}^{\mu, \varepsilon}, \eta> & \\
= & \mathbf{E}^{\dagger}\left[\eta\left(x_{k}^{\varepsilon}\right) \exp \left(\frac{\mu}{\varepsilon} \sum_{l=1}^{k} L\left(x_{l}^{\varepsilon}, w_{l}^{\varepsilon}, u_{l-1}\right)\right) \mathcal{Z}_{k}^{\varepsilon} \mid \mathcal{Y}_{k}\right] \\
= & \mathbf{E}^{\dagger}\left[\eta\left(x_{k}^{\varepsilon}\right) \exp \left(\frac{\mu}{\varepsilon} L\left(x_{k}^{\varepsilon}, w_{k}^{\varepsilon}, u_{k-1}\right)\right) \Psi^{\varepsilon}\left(x_{k-1}^{\varepsilon}, y_{k}^{\varepsilon}\right)\right. \\
& \left.\exp \left(\frac{\mu}{\varepsilon} \sum_{l=1}^{k-1} L\left(x_{l}^{\varepsilon}, w_{l}^{\varepsilon}, u_{l-1}\right)\right) \mathcal{Z}_{k-1}^{\varepsilon} \mid \mathcal{Y}_{k}\right] \\
= & \mathbf{E}^{\dagger}\left[\int_{\boldsymbol{R}^{n}} \int_{F\left(x_{k-1}, u_{k-1}\right)} \eta(z)\right. \\
& \exp \left(\frac{\mu}{\varepsilon} L\left(z, z-r, u_{k-1}\right)\right) \Psi^{\varepsilon}\left(x_{k-1}^{\varepsilon}, y_{k}^{\varepsilon}\right) . \\
& \chi\left(x_{k-1}, u_{k-1}\right) \exp \left(\frac{\mu}{\varepsilon} \sum_{l=1}^{k-1} L\left(x_{l}^{\varepsilon}, w_{l}^{\varepsilon}, u_{l-1}\right)\right) \\
& \left.\mathcal{Z}_{k-1}^{\varepsilon} \psi^{\varepsilon}(z-r) d r d z \mid \mathcal{Y}_{k}\right] \\
=< & \sigma_{k-1}^{\varepsilon}, \int_{R^{n}} \int_{F\left(\cdot, u_{k-1}\right)} \eta(z) \\
& \exp \left(\frac{\mu}{\varepsilon} L\left(z, z-r, u_{k-1}\right)\right) \chi\left(\cdot, u_{k-1}\right) . \\
& \Psi^{\varepsilon}\left(\cdot, y_{k}^{\varepsilon}\right) \psi^{\varepsilon}(z-r) d r d z> \\
=< & \sigma_{k-1}^{\varepsilon}, \Sigma^{\mu, \varepsilon}\left(u_{k-1}, y_{k}^{\varepsilon}\right) \eta> \\
=< & \Sigma^{\mu, \varepsilon *}\left(u_{k-1}, y_{k}^{\varepsilon}\right) \sigma_{k-1}^{\varepsilon}, \eta>
\end{aligned}
$$

for any $\eta \in L^{\infty}\left(\boldsymbol{R}^{n}\right)$.

Observe that for all $u \in U_{0, K-1}$, we have

$$
\begin{aligned}
\mathbf{E}^{\dagger}[ & \left.<\sigma_{K}^{\mu, \varepsilon}, 1>\right] \\
& =\mathbf{E}^{\dagger}\left[\mathbf{E}^{\dagger}\left[\exp \left(\frac{\mu}{\varepsilon} \sum_{l=1}^{K} L\left(x_{l}^{\varepsilon}, w_{l}^{\varepsilon}, u_{l-1}\right)\right) \mathcal{Z}_{K}^{\varepsilon} \mid \mathcal{Y}_{K}\right]\right] \\
& =\mathbf{E}^{\dagger}\left[\exp \left(\frac{\mu}{\varepsilon} \sum_{l=1}^{K} L\left(x_{l}^{\varepsilon}, w_{l}^{\varepsilon}, u_{l-1}\right)\right) \mathcal{Z}_{K}^{\varepsilon}\right] \\
& =J^{\mu, \varepsilon}(\rho, u)
\end{aligned}
$$

Thus, the cost can be expressed as a function of $\sigma_{K}^{\mu, \varepsilon}$ alone, and hence the name information state for $\sigma_{k}^{\mu, \varepsilon}$ is justified. We can now obtain the solution to the risk-sensitive stochastic control problem via dynamic programming. This methodology is well known in the stochastic control literature [5],[4]. Define the value function for $\sigma \in L^{1}\left(\boldsymbol{R}^{n}\right)$ by

$$
V^{\mu, \varepsilon}(\sigma, k)=\inf _{u \in U_{k, K-1}} \mathbf{E}^{\dagger}\left[<\sigma_{K}^{\mu, \epsilon}, 1>\mid \sigma_{k}^{\mu, \varepsilon}=\sigma\right]
$$


lem. We also present necessary and sufficient conditions for the solvability of the deterministic problem. These conditions are derived independently of section 2. Thus, once the structure of the controller has been discovered by taking small noise limits to the risk-sensitive stochastic control problem, the actual deterministic results can be derived directly with less restrictive assumptions and easier mathematical arguments.

\section{Risk-Sensitive Stochastic Control}

We will consider a special case of the risk sensitive stochastic control problem. On a probability space $\left(\Omega, \mathcal{F}, \mathbf{P}^{u}\right)$ consider the stochastic control problem

$$
\begin{aligned}
& x_{k+1}^{\varepsilon}=\xi_{k}^{\varepsilon}+w_{k+1}^{\varepsilon}, \xi_{k}^{\varepsilon} \in F\left(x_{k}^{\varepsilon}, u_{k}\right) \\
& y_{k+1}^{\varepsilon}=\nu_{k}^{\varepsilon}+v_{k+1}^{\varepsilon}, \nu_{k}^{\varepsilon} \in G\left(x_{k}^{\varepsilon}\right)
\end{aligned}
$$

on the finite time interval $k=0, \ldots, K-1$. The process $y^{\varepsilon} \in \boldsymbol{R}$ is measured, and is called the observation process. $x^{\varepsilon} \in \boldsymbol{R}^{n}$ represent the states. For convenience, we will write the dynamics as

$$
\begin{aligned}
& x_{k+1}^{\varepsilon} \in F\left(x_{k}^{\varepsilon}, u_{k}\right)+w_{k+1}^{\varepsilon} \\
& y_{k+1}^{\varepsilon} \in G\left(x_{k}^{\varepsilon}, u_{k}\right)+v_{k+1}^{\varepsilon}
\end{aligned}
$$

Denote by $s_{k, k+j}$, the sequence $\left\{s_{k}, s_{k+1}, \ldots, s_{k+j}\right\}$. Let $\mathcal{G}_{k}, \mathcal{Y}_{k}$ denote the complete filtrations generated by $\left(x_{0, k}^{\varepsilon}, y_{0, k}^{\varepsilon}\right)$ and $y_{0, k}^{\varepsilon}$ respectively. We assume

A1. $y_{0}^{\varepsilon}=0$

A2. $\left\{w_{k}^{\varepsilon}\right\}$ is a $\boldsymbol{R}^{n}$-valued i.i.d. noise sequence with density $\psi^{\varepsilon}(w)=(2 \pi \varepsilon)^{-n / 2} \exp \left(-\frac{1}{2 \varepsilon}|w|^{2}\right)$.

A3. $\left\{v_{k}^{\varepsilon}\right\}$ is a real-valued i.i.d. noise sequence with density

$\phi^{\varepsilon}(v)=(2 \pi \varepsilon)^{-1 / 2} \exp \left(-\frac{1}{2 \varepsilon}|v|^{2}\right)$, independent of $\left\{w_{k}^{\varepsilon}\right\}$.

A4. $\left\{\xi_{k}^{\varepsilon}\right\}$ is a $\boldsymbol{R}^{n}$-valued random sequence with $\xi_{k}^{\varepsilon} \in$ $F\left(x_{k}^{\varepsilon}, u_{k}\right)$, having a uniform density $\chi\left(x_{k}^{\varepsilon}, u_{k}\right)=\left(\int_{\xi \in F\left(x_{k}^{c}, u_{k}\right)} d \xi\right)^{-1}$ for each $k$. Furthermore, for each $k, \xi_{k}^{\varepsilon}$ is independent of $w_{l}^{\varepsilon}$ and $v_{l}^{\varepsilon}, l=k+1, \ldots, K$. Similarly, $\left\{\nu_{k}^{\varepsilon}\right\}$ is a $R$ valued random sequence with $\nu_{k}^{\varepsilon} \in G\left(x_{k}^{\varepsilon}\right)$ having a uniform density $\theta\left(x_{k}^{\varepsilon}\right)=\left(\int_{\nu \in G\left(x_{k}^{c}\right)} d \nu\right)^{-1}$ for each $k$. Furthermore, for each $k, \nu_{k}^{\varepsilon}$ is independent of $\xi_{l}^{\varepsilon}, w_{l+1}^{\varepsilon}, v_{l+1}^{\varepsilon}$ for $l=k, \ldots, K-1$.

A5. The controls $u_{k}$ take values in $U \subset \boldsymbol{R}^{m}$ assumed compact and are $\mathcal{Y}_{k}$ measurable.

A6. $F$ is a set-valued map from $\boldsymbol{R}^{n} \times \boldsymbol{R}^{m}$ to $\boldsymbol{R}^{n}$, uniformly continuous in $x$, uniformly in $u \in U$. $G$ is a set-valued map from $\boldsymbol{R}^{n}$ to $\boldsymbol{R}$, satisfying the same assumptions as $F$.
A7. Furthermore, $F, G$ assume convex compact values and have a non-empty interior for all $x$ and $u$. $\chi, \theta$ are uniformly continuous in $x$, uniformly in $u \in U$, and are bounded.

A8. $x_{0}^{\varepsilon}$ has density $\rho(x)=(2 \pi)^{-n / 2} \exp \left(\frac{-1}{2}|x|^{2}\right)$.

We could let $\eta_{k}^{\varepsilon}$, and $\nu_{k}^{\varepsilon}$ be generated by other (nonuniform) densities. However, the uniform density assumption allows simplification of the mathematical development. Note that assumption A7 places restrictions on $F$ and $G$. An example of $F$ which satisfies these assumptions is

$$
F(x, u)=A x+B u+\overline{\mathcal{B}}_{r}(0)
$$

where, $A$, and $B$ are matrices of appropriate dimensions, and $\overline{\mathcal{B}}_{r}(0)$ is the closed ball of radius $r$, centered at 0 . Note that $\xi_{k}^{\varepsilon}, \nu_{k}^{\varepsilon}$ will in general depend on all the past values of $w^{\varepsilon}$, and $v^{\varepsilon}$, through the state $x_{k}^{\varepsilon}$, and control $u_{k}$. At time $k$, let $U(k)$ denote the set of control functions $u_{k}$ which satisfy A5, i.e. $u_{k}$ take values in $U$, and are a function of $y_{0, k}^{\varepsilon}$. For $l \geq 0$, we write $U_{k, k+l}=U(k) \bigcup U(k+1) \cdots \bigcup U(k+l)$. For $\mu>0$, the cost function for the risk-sensitive stochastic control problem is defined for admissible $u \in U_{0, K-1}$ by

$$
J^{\mu, \varepsilon}(\rho, u)=\mathbf{E}^{u}\left[\exp \left(\frac{\mu}{\varepsilon} \sum_{k=1}^{K} L\left(x_{k}^{\varepsilon}, w_{k}^{\varepsilon}, u_{k-1}\right)\right)\right]
$$

and the partially observed risk-sensitive stochastic control problem is to find $u^{*} \in U_{0, K-1}$ such that

$$
J^{\mu, \varepsilon}\left(\rho, u^{*}\right)=\inf _{u \in U_{0, K-1}} J^{\mu, \varepsilon}(\rho, u)
$$

We further assume that

A9. $L \in C\left(\boldsymbol{R}^{n} \times \boldsymbol{R}^{n} \times \boldsymbol{R}^{m}\right)$ is single-valued, nonnegative, bounded and uniformly continuous.

\subsection{Change of Measure}

Using an idea from [4], suppose there exists a reference measure $\mathbf{P}^{\dagger}$ such that under $\mathbf{P}^{\dagger},\left\{y_{k}^{\varepsilon}\right\}$ is i.i.d. with density $\phi^{\varepsilon}$, independent of $\left\{x_{k}^{\varepsilon}\right\}$ where $x^{\varepsilon}$ satisfies

$$
x_{k+1}^{\varepsilon} \in F\left(x_{k}^{\varepsilon}, u_{k}\right)+w_{k+1}^{\varepsilon}
$$

Define

$$
\Lambda_{k}^{\varepsilon}=\Pi_{l=1}^{k}\left(\theta\left(x_{l-1}^{\varepsilon}\right) \int_{G\left(x_{l-1}^{\varepsilon}\right)} \phi^{\varepsilon}\left(v_{l}^{\varepsilon}+\xi\right) d \xi / \phi^{\varepsilon}\left(v_{l}^{\varepsilon}\right)\right) .
$$

and define $\mathbf{P}^{\dagger}$ by setting

$$
\left.\frac{d \mathbf{P}^{\dagger}}{d \mathbf{P}^{u}}\right|_{\mathcal{G}_{k}}=\Lambda_{k}^{\varepsilon}
$$

i.e. by setting the Radon-Nikodym derivative, restricted to $\mathcal{G}_{k}$ to equal $\Lambda_{k}^{\varepsilon}$. Note that in general $\mathbf{P}^{\dagger}$ at $k$, may depend on the states $x_{0, k-1}^{\varepsilon}$ (but not on $x_{k}^{\varepsilon}$ ), however we hide this to prevent notational clutter. We write $\mathbf{E}^{\dagger}, \mathbf{E}^{u}$ to denote expectations with respect to the measures $\mathbf{P}^{\dagger}, \mathbf{P}^{u}$ respectively. Then 
$k=0, \ldots, K-1 ;$ where $\bar{u}_{k}^{*}(p)$ achieves the minimum in (9), $p_{0}=\delta_{0}$, and let $p$ be the corresponding information state trajectory with $p_{k} \in$ dom $M_{K-k}$, $k=1, \ldots, K$. Then $u^{*}$ solves the finite time output feedback robust control problem with the initial condition $x_{0}=0$.

\subsection{A Note on Feasible States}

For the remainder of the paper, we lift the restriction that $x_{0}=0$, and let it be arbitrary, but known. An interesting property of the information state is that it also acts as an indicator function for feasible states. Definition: For a given initial state $x_{0}$, an output trajectory $y_{1, k+1}$, and a control trajectory $u_{0, k}$, a state $\bar{x}_{k+1}$ is called feasible at time $k+1$ if there exists a state trajectory $x_{0, k+1}$ with $x_{k+1}=\bar{x}_{k+1}$ such that $x_{j+1} \in F\left(x_{j}, u_{j}\right)$ and $y_{j+1} \in G\left(x_{j}\right)$ for $j=0, \ldots, k$.

Consider the following recursion

$$
\begin{aligned}
& \mathcal{X}_{k+1}^{y, u}\left(x_{0}\right)=F\left(G^{-1}\left(y_{k+1}\right) \cap \mathcal{X}_{k}^{y, u}\left(x_{0}\right), u_{k}\right) \\
& \mathcal{X}_{0}^{y, u}\left(x_{0}\right)=\left\{x_{0}\right\}
\end{aligned}
$$

for $k=0, \ldots, K-1$, where $G^{-1}\left(y_{k+1}\right)=\left\{x \in \boldsymbol{R}^{n} \mid\right.$ $\left.y_{k+1} \in G(x)\right\}$, and for a set $M \subset \boldsymbol{R}^{n}$, we define $F(M, u)=\bigcup_{x \in M} F(x, u)$. Define the following limiter function $\tau(\cdot): \boldsymbol{R}_{+} \rightarrow \boldsymbol{R}^{-}$as

$$
\tau(x) \triangleq \begin{cases}0 & \text { if } x \geq 0 \\ x & \text { else }\end{cases}
$$

where $\boldsymbol{R}_{+}$denotes the extended real line and $\boldsymbol{R}^{-}$denotes $\left\{x \in \boldsymbol{R}_{+} \mid x \leq 0\right\}$.

Theorem 4 Suppose $p_{0}=\delta_{\left\{x_{0}\right\}}$ then

$$
\tau\left(p_{k}(x)\right)=\delta_{\mathcal{X}_{k}^{y, u}\left(x_{0}\right)}(x), \forall x \in \boldsymbol{R}^{n}
$$

Remark: Thus, we see that the information state can be transformed by a simple limiting operation to the indicator function of the set of feasible states. This has implications on the computability of the problem, as suppose the system starts from rest (i.e. $x_{0}=0$ ). Then, clearly by lemma 3 , the information state is always nonpositive. Hence, it is zero on feasible states, and $-\infty$ elsewhere. Thus, instead of computing the information state via (6), one could consider propagating the set of feaible states (the so called problem of guaranteed estimation $[7],[6])$.

\section{Appendix}

Here, we state an extension of the Varadhan-Laplace lemma presented in [5]. Below $\rho$ denotes a metric on $C\left(\boldsymbol{R}^{n} \times \boldsymbol{R}^{p}\right)$ corresponding to uniform convergence on compact subsets. $\mathcal{B}_{r}(x)$ denotes the open ball centered at $x$ of radius $r$, and $G^{a}$ is a set-valued map, $G^{a}: \boldsymbol{R}^{n} \rightarrow \boldsymbol{R}^{p}$
Lemma 4 Let $A$ be a compact space, $F_{a}^{\varepsilon}$, $F_{a} \in C\left(\boldsymbol{R}^{n} \times \boldsymbol{R}^{p}\right)$ and assume

i. $\lim _{\varepsilon \rightarrow 0} \sup _{a \in A} \rho\left(F_{a}^{\varepsilon}, F_{a}\right)=0$

ii. The function $F_{a}$ is uniformly continuous in each argument on each set $\mathcal{B}_{R}(0) \times \mathcal{B}_{\hat{R}}(0) ; R, \hat{R}>0$, uniformly in $a \in A$.

iii. $\exists \gamma_{1}>0, \gamma_{2} \geq 0$ such that

$$
\begin{aligned}
& F_{a}^{\varepsilon}(x, w), F_{a}(x, w) \leq-\gamma_{1}\left(|x|^{2}+|w|^{2}\right)+\gamma_{2} \\
& \forall x \in \boldsymbol{R}^{n}, \forall w \in \boldsymbol{R}^{m}, \forall a \in A, \forall \varepsilon>0 .
\end{aligned}
$$

$i v . G^{a}$ is uniformly continuous with convex compact values on each set $\mathcal{B}_{R}(0)$, uniformly in $a \in$ A.

v. Int $G^{a}(x) \neq \phi, \forall x \in \boldsymbol{R}^{n}, \forall a \in A$.

Then

$$
\begin{array}{r}
\lim _{\varepsilon \rightarrow 0} \sup _{a \in A} \mid \varepsilon \log \int_{\boldsymbol{R}^{n}} \int_{G^{a}(x)} e^{F_{a}^{\varepsilon}(x, w) / \varepsilon} d w d x- \\
\sup _{x \in \boldsymbol{R}^{n}} \sup _{w \in G^{a}(x)} F_{a}(x, w) \mid=0
\end{array}
$$

\section{References}

[1] J.S. Baras and N.S. Patel. Robust control of setvalued dynamical systems. ISR Technical Report (TR 94-75) also submitted to IEEE Transactions on Automatic Control.

[2] J.S. Baras and N.S. Patel. Robust control of discrete time generalized dynamical systems: Finite and infinite time results. In Proc. of the American Control Conference, pages 1990-1994, 1995.

[3] A. Bensoussan and J.H. van Schuppen. Optimal control of partially observed stochastic systems with an exponential-of-integral performance index. SIAM J. Control and Optimization, 23(4):599-613, 1985.

[4] R.J. Elliott and J.B. Moore. Discrete time partially observed control. In D. Elworthy, W.N. Everitt, and E.B. Lee, editors, Differential Equations, Dynamical Systems and Control Science, volume 32. Marcel Dekker, 1993.

[5] M.R. James, J.S. Baras, and R.J. Elliott. Risksensitive control and dynamic games for partially observed discrete-time nonlinear systems. IEEE Transactions on Automatic Control, 39(4):780$792,1994$.

[6] A.B. Kurzhanski. Identification - a theory of guaranteed estimates. In J. Willems, editor, From Data to Model. Springer-Verlag, 1988.

[7] F.C. Schweppe. Uncertain Dynamical Systems. Prentice Hall, 1973. 